\title{
Impaired corneal sensation and nerve loss in a type 2 rat model of chronic diabetes is reversible with combination therapy of menhaden oil, a-lipoic acid and enalapril
}

\author{
Eric P. Davidson ${ }^{\mathrm{a}}$, Lawrence J. Coppey ${ }^{\mathrm{a}}$, Hanna Shevalye ${ }^{\mathrm{a}}$, Alexander Obrosov ${ }^{\mathrm{a}}$, Randy H. \\ Kardon, MD, PhD ${ }^{b, c, d}$, and Mark A. Yorek, PhD ${ }^{a, b, c, e}$ \\ aDepartment of Internal Medicine, University of lowa, lowa City, IA, 52242 USA \\ ${ }^{b}$ Department of Veterans Affairs lowa City Health Care System, lowa City, IA, 52246 USA \\ 'Veterans Affairs Center for the Prevention and Treatment of Visual Loss, lowa City, IA, 52246 \\ USA \\ dDepartment of Ophthalmology and Visual Sciences, University of lowa, lowa City, IA, 52242 USA \\ eFraternal Order of Eagles Diabetes Research Center, University of lowa, lowa City, IA, 52242 \\ USA
}

\section{Abstract}

Purpose-This study investigated the efficacy of monotherapy vs. combination of menhaden oil, a-lipoic acid, and enalapril on cornea sensation and morphometry and other neuropathy related endpoints in a rat model of type 2 diabetes.

\begin{abstract}
Methods-Male Sprague-Dawley rats, 12 weeks of age, were fed a high fat diet for 8 weeks followed by $30 \mathrm{mg} / \mathrm{kg}$ streptozotocin. After sixteen weeks of hyperglycemia, 12 week treatments consisting of menhaden oil, a-lipoic acid, enalapril or their combination were initiated. Prior to and after treatments we performed analyses of multiple neural and vascular endpoints including cornea sensitivity, corneal nerve density, vascular reactivity of epineurial arterioles, motor and sensory nerve conduction velocity, intraepidermal nerve fiber density and thermal nociception.
\end{abstract}

Results-Prior to treatment all the neural and vascular endpoints in diabetic rats were impaired. Treating diabetic rats with monotherapy was effective in improving neural and vascular deficits with menhaden oil being most efficacious. However, the combination therapy provided the greatest benefit and improved/reversed all nerve and vascular deficits. The effect of combination therapy on cornea relative sensitivity (AUC) and structure $\left(\mathrm{mm} / \mathrm{mm}^{2}\right)$, primary endpoints for this study, for control, diabetic, and diabetic treated rats was $4.2 \pm 1.4$ and $7.5 \pm 0.5,12.1 \pm 1.3^{*}$ and $3.8 \pm 0.2^{*}$, $6.6 \pm 2.3^{+}$and $7.3 \pm 0.5^{+}$, respectively $(* \mathrm{p}<0.05$ compared to control $; \mathrm{p}<0.05$ compared to diabetic).

Conclusions-These studies suggest that a combination therapeutic approach may be most effective for treating vascular and neural complications of type 2 diabetes.

\footnotetext{
Correspondence should be addressed to: Mark A. Yorek, Room 204, Building 40, Department of Veterans Affairs Iowa City Health Care System, Iowa City, IA 52246, Tel \#: 1-319-338-0581 ext. 7696, Fax \# 1-319-339-7162, mark-yorek@uiowa.edu.
} 


\section{Keywords}

diabetes; corneal confocal microscopy; neuropathy; corneal sensitivity

\section{Introduction}

Diabetic peripheral neuropathy is a major and devastating complication of diabetes. The search for an effective treatment has been challenging in part due to its complex etiology. Diabetic peripheral neuropathy has been described by some investigators to be a disease of the vasculature leading to nerve ischemia and altered nerve function. Other investigators have proposed that diabetic peripheral neuropathy is caused by a combination of metabolic defects. Additional contributors to diabetic peripheral neuropathy have been reported to include increased formation of advanced glycation endproducts, reduced neurotrophic support and increased inflammatory and oxidative stress. Overall, these mechanisms and likely others cause damage to neurons, Schwann cells and the vasculature. Ultimately, relentless damage to the nerve complex and surrounding vasculature leads to diabetic peripheral neuropathy. Given the complex etiology of diabetic peripheral neuropathy, a successful treatment will likely require a combination of early detection, life-style changes and pharmaceutical interventions targeting the mechanisms deemed most responsible for the pathogenesis.

In pre-clinical studies using diabetic rats we have found that mono-therapies consisting of enalapril, a-lipoic acid or menhaden oil are effective in improving peripheral neuropathy and/or vascular impairment of epineurial arterioles of the sciatic nerve. ${ }^{1,2}$ However, these treatments were incapable of fully preventing diabetes-induced nerve or vascular damage. When we combined these three compounds in studies using a type 2 diabetic rat model and an early intervention protocol we found the combination to be more efficacious. ${ }^{1}$ In this study, we investigated the individual and combined effect of these same compounds following a late intervention to test for reversibility and return of function. Treatments were not initiated until after development of significant vascular and neural impairment, including decrease in corneal sensation and loss of sub-epithelial corneal nerves. Thus, the study design allowed us to evaluate the reversibility of diabetes induced peripheral neuropathy with an emphasis on corneal function and structure.

\section{Methods}

Unless stated otherwise all chemicals used in these studies were obtained from Sigma Aldrich Chemical Co. (St. Louis, MO).

\section{Animals}

Sprague-Dawley (Harlan Sprague Dawley, Indianapolis, IN) male rats 10-11 weeks of age were housed in a certified animal care facility and food (Harlan Teklad, \#7001, Madison, WI) and water were provided ad libitum. All institutional (ACORP \#1590601) and NIH guidelines for use of animals were followed. The studies also adhered to the Association for Research in Vision and Ophthalmology (ARVO) statement for the use of animals in 
research. At 12 weeks of age, rats were separated into two groups and fed a control diet (Harlan Teklad, \#7001, 4.25\% kcal as fat, $3.0 \mathrm{kcal} / \mathrm{g}$, Madison, WI) or a high fat diet (D12451 (45\% kcal as fat, $4.7 \mathrm{kcal} / \mathrm{g}$ ); Research Diets, New Brunswick, NJ). Rats were maintained on these diets for 8 weeks and then high fat fed rats were treated with streptozotocin (30 mg/kg in 0.1M citric acid buffer, $\mathrm{pH} 4.5$, i.p., EMD/Millipore, Billerica, MA) to induce hyperglycemia. Control fed rats was treated with vehicle. Blood glucose was evaluated 96h later using glucose-oxidase reagent strips (Aviva Accu-Chek, Roche, Mannheim, Germany) and rats having blood glucose level of $250 \mathrm{mg} / \mathrm{dl}(13.8 \mathrm{mM})$ or greater were considered to be diabetic. ${ }^{3}$ Control and diabetic rats were maintained on their respective diets for an additional sixteen weeks. Afterwards, the diabetic rats were divided into five groups. Based on power calculations using corneal nerve sensitivity and density as the primary endpoints it was determined an $\mathrm{n}$ of 10 rats would be required for each group. One group was designated as the untreated group. The other four groups received the $45 \mathrm{kcal}$ $\%$ high fat diet containing: menhaden oil ( $1 / 2$ of the kcal of fat in the $45 \mathrm{kcal} \%$ high fat diet was substituted with menhaden oil), or enalapril $(500 \mathrm{mg} / \mathrm{kg}$ in the $45 \mathrm{kcal} \%$ high fat diet (BOC Sciences, Shirley, NY)), or a-lipoic acid ( $2.5 \mathrm{~g} / \mathrm{kg}$ in the $45 \mathrm{kcal} \%$ high fat diet) or the combination of all three. The modified diets were prepared by Research Diets. The doses of the agents used in the modified diets was based on a previous study. ${ }^{1}$ These diets (treatment phase) were maintained for 12 weeks. We examined a set of control and diabetic rats at the time treatment began to establish a baseline for the pathology present after 24 weeks of high fat diet and 16 weeks of untreated hyperglycemia.

\section{Glucose tolerance}

Glucose tolerance was determined by injecting rats with a saline solution containing $2 \mathrm{~g} / \mathrm{kg}$ glucose, i.p., after an overnight fast as previously described. ${ }^{1}$

\section{Thermal nociceptive response and corneal reactivity}

Thermal nociceptive response in the hindpaw was measured using the Hargreaves method as previously described. ${ }^{3}$ Data was reported in seconds. Corneal sensation was measured using a Cochet-Bonnet filament esthesiometer in un-anaesthetized rats (Luneau Ophtalmologie, France) as previously described. ${ }^{4}$ The data was reported in $\mathrm{cm}$ of filament length.

Corneal sensation was also measured by applying buffered isotonic and hypertonic eye drops ( 290 and $900 \mathrm{mOsm} / \mathrm{liter}$ ) to un-anaesthetized rats as previously described. ${ }^{5}$ Animals were placed in a custom made restraining apparatus and six CMOS cameras (Imaging Development Systems GmbH, Obersulm, Germany), three per each eye, were positioned in order to observe both eyes simultaneously. Custom software was used to synchronize video streams and obtain images (MATLAB R2012a, The MathWorks Inc., Natick, MA). Video recording was started $30 \mathrm{sec}$ before the first epoch. Images from this recording period were used to establish a baseline area of eyelid opening. The first recording epoch began with the addition of $20 \mu \mathrm{l}$ of the isotonic solution to the right eye. The recording period was for $3 \mathrm{~min}$ followed by a 5 min recovery period. The next epoch began with the addition of the hypertonic solution. An image collector was used offline to retrieve video frames from each epoch. Fiji image analysis software was used by a masked technician to measure the visible surface area of both eyes between the upper and lower eyelids. These areas were expressed 
as a percentage of the baseline area of eyelid opening. These data were plotted vs. time and the area under the curve used to quantify corneal sensitivity to topical isotonic and hypertonic saline, based on narrowing of the palpebral fissure in response to corneal nerve reactivity.

\section{Corneal innervation}

On the day of the terminal studies, rats were weighed and anesthetized with Nembutal (50 mg/kg, i.p., Abbott Laboratories, North Chicago, IL). Sub-epithelial corneal nerves were imaged using the Rostock cornea module of the Heidelberg Retina Tomograph confocal microscope as previously described. ${ }^{4}$ The investigator acquiring these images was masked with respect to identity of the animal condition. Corneal nerve fiber length was defined as the total length of all nerve fibers and branches (in millimeters) present in the acquired images standardized for area of the image (in square millimeters). ${ }^{4}$ The corneal fiber length for each animal was the mean value obtained from the acquired images and expressed as $\mathrm{mm} / \mathrm{mm}^{2}$.

\section{Motor and sensory nerve conduction velocity}

Motor nerve conduction velocity was determined as previously described using a noninvasive procedure in the sciatic-posterior tibial conducting system. ${ }^{3}$ Sensory nerve conduction velocity was determined using the digital nerve as described by Obrosova et al. ${ }^{6}$ Motor and sensory nerve conduction velocities were reported in meters per second.

\section{Vascular reactivity in epineurial arterioles}

Videomicroscopy was used to investigate in vitro vasodilatory responsiveness of epineurial arterioles vascularizing the region of the sciatic nerve as previously described. ${ }^{1}$ Following isolation and suspension of the vessels cumulative concentration-response relationships were evaluated for acetylcholine $\left(10^{-8}-10^{-4} \mathrm{M}\right)$. At the end of each dose response curve for acetylcholine papaverine $\left(10^{-5} \mathrm{M}\right)$ was added to determine maximal vasodilation.

\section{Intraepidermal nerve fiber density in the hindpaw}

Immunoreactive nerve fiber profiles innervating the skin from the hindpaw were visualized using standard confocal microscopy as previously described. ${ }^{3}$ Profiles were counted by two individual investigators that were masked to the sample identity. All immunoreactive profiles were counted and normalized to length and the data are presented as profiles $/ \mathrm{mm}$.

\section{Physiological markers}

Non-fasting blood glucose was determined with Aviva Accu-Chek strips. Serum was collected for determining levels of free fatty acid, triglyceride, free cholesterol, adiponectin and activity of angiotensin converting enzyme using commercial kits from Roche Diagnostics, Mannheim, Germany; Sigma Chemical Co., St. Louis, MO; Bio Vision, Mountain View, CA; Alpco, Salem, NH; and Buhlmann Laboratories AG, Schonenbuch, Switzerland, respectively. Serum was also used to determine thiobarbituric acid reactive substances as described by Mihara et al. and modified by Siman and Eriksson. 7,8 


\section{Data Analysis}

Results are presented as mean \pm S.E.M. as in figure legends and tables. Comparisons between groups were conducted using a one-way ANOVA and Bonferroni's test for multiple comparisons (Prism software; GraphPad, San Diego, CA). A p value of less than 0.05 was considered significant.

\section{Results}

In Table 1 (see supplemental data) data are provided for the change in weight, diet consumption and blood glucose for control and diabetic rats in the treatment paradigm. At the beginning of the study all rats weighed about the same. After 24 weeks of the high fat diet and 16 weeks post streptozotocin treatment, all diabetic rats were randomized into 5 groups with 10 rats per group. Prior to initiation of treatment, all diabetic rats in each of the groups weighed less than the control rats. The difference was significant for three of the groups: untreated diabetic, diabetic to be treated with menhaden oil and diabetic to be treated with the combination of menhaden oil, a-lipoic acid and enalapril. During the 12 week treatment period 5 diabetic rats $(10 \%)$ were lost to the study. Three developed nodules and were sacrificed. One became lame and loss use of the right leg and was sacrificed and the other loss was due to sudden unexplained death. Data from these 5 rats were excluded.

During the treatment phase we monitored diet consumption for all groups. Data in Table 1 (see supplemental data) demonstrate that control rats consumed less chow than the untreated and treated diabetic rats but the difference was not significant. All diabetic rats consumed about the same amount of chow throughout the study suggesting that the amount of menhaden oil, a-lipoic acid and/or enalapril received was constant during the treatment phase.

At the end of the study all diabetic rats weighed significantly less than control rats. Nonfasting blood glucose levels were significantly increased in all diabetic rats compared to control rats at the beginning of the study and this was not changed with any treatment. Data in Figure 1 (see supplemental data) demonstrate that glucose clearance was significantly impaired in diabetic rats prior to treatment compared to control rats. At the end of the treatment phase glucose clearance remained impaired in untreated diabetic rats and this was not significantly improved by the mono- or combination therapies (Figure 1).

Table 1 provides data for serum lipid, thiobarbituric acid reactive substances (TBARS) and adiponectin levels and angiotensin converting enzyme activity at the end of the treatment phase. Serum free fatty acid, triglyceride and cholesterol levels as well as TBARS were all significantly increased in untreated diabetic rats. Mono-therapy with menhaden oil, a-lipoic acid or enalapril lowered serum lipid levels and TBARS. However, combination therapy was the most effective and completely corrected serum lipid and TBARS levels. Levels of adiponectin in serum were unchanged in untreated diabetic rats. Treating diabetic rats with menhaden oil, a-lipoic acid or enalapril trended to increase serum adiponectin levels but this was not significant. However, treating diabetic rats with the combination of menhaden oil, a-lipoic acid and enalapril significantly increased serum adiponectin levels. As expected, diabetes significantly increased serum angiotensin converting enzyme activity and this 
activity was significantly decreased by treatment with enalapril or the combination of menhaden oil, a-lipoic acid and enalapril.

We have previously demonstrated that impaired vascular reactivity of epineurial arterioles of the sciatic nerve preceded slowing of nerve conduction velocity. ${ }^{9}$ Data in Figure 2 (see supplemental data) demonstrate that prior to initiation of treatments vascular relaxation to acetylcholine by epineurial arterioles was significantly impaired. Following the treatment phase vascular relaxation to acetylcholine was further decreased in untreated diabetic rats (Figure 2). Mono-therapy with menhaden oil, a-lipoic acid or enalapril significantly improved vascular relaxation to acetylcholine compared to untreated diabetic rats. However, mono-therapy treatment did not fully restore vascular reactivity. In contrast, treating diabetic rats with the combination of menhaden oil, a-lipoic acid and enalapril totally reversed the diabetes-induced vascular dysfunction.

The effect of treating chronic diabetic rats with menhaden oil, a-lipoic acid, enalapril or their combination on neural function related endpoints are provided in Table 2 and Figure 3. Prior to the initiation of treatment diabetic rats had slowed motor and sensory nerve conduction velocity, thermal hypoalgesia, decrease in intraepidermal nerve fibers in the skin of the hindpaw, impaired corneal sensitivity, as determined by Cochet-Bonnet filament esthesiometer, and decrease in corneal fiber length (Table 2 see baseline control and baseline diabetic columns). Representative images of sub-epithelial nerves from the cornea of a control and untreated diabetic rat are provided in Figure 3 (see supplemental data). Cornea function as determined by evaluating sensitivity to a hypertonic solution was also impaired in diabetic rats prior to treatment (Figure 3 see basal data). The increase in area under the curve, graphed as relative cornea sensitivity, represents a decrease in squinting in response to the hypertonic solution and thus a decrease in corneal sensitivity. In this study and as previously reported there was no response when an isotonic solution was applied to the cornea and the untreated left eye remained unresponsive throughout the study. ${ }^{5}$

There was a trend for the neural endpoints to exacerbate in untreated diabetic rats during the 12 week treatment phase. In general treating chronically diabetic rats with menhaden oil as a mono-therapy was more effective than treatment with either a-lipoic acid or enalapril on neural endpoints and cornea sensitivity and structure and for some endpoints was equivalent to the combination therapy. However, overall we found that combination therapy consisting of menhaden oil, a-lipoic acid and enalapril was the most efficacious in improving diabetic peripheral neuropathy including restoration of corneal nerve sensitivity and structure and vascular reactivity.

\section{Discussion}

Determining corneal nerve sensitivity and sub-epithelial nerve density is being promoted as a surrogate marker and method for early diagnosis of diabetic peripheral neuropathy. ${ }^{10,11}$ Other than two studies demonstrating corneal nerve regeneration one year post pancreas or pancreas/kidney transplant, little is known about the ability of corneal nerves to regenerate and regain functional sensitivity once nerve function and structure are compromised by 
chronic untreated diabetes and/or serve as a biomarker of recovery for other diabetic neuropathy endpoints. ${ }^{12,13}$

The goal of this study was to determine whether optimal treatment conditions could restore corneal nerve density and functional sensitivity as well as other diabetic neuropathy endpoints once significantly impacted by chronic hyperglycemia. For these studies we used a rat model of type 2 diabetes. ${ }^{3}$ Interventions were started after 24 weeks of a high fat diet and 16 weeks of hyperglycemia. The treatment paradigm included mono-therapy of optimal doses of menhaden oil, a-lipoic acid or enalapril as well as the combination of all three compounds. ${ }^{1}$ Previously we have reported that these three compounds individually had beneficial effects on diabetic peripheral neuropathy and each have also been shown to be safe for use in humans. ${ }^{1,3,14-18}$ Furthermore, we have shown that combining these three compounds was more efficacious as a treatment for diabetic peripheral neuropathy than mono-therapy. ${ }^{1}$ However, this previous study was done using an early intervention protocol and was designed to determine whether treatments could slow progression of diabetic peripheral neuropathy. ${ }^{1}$ The protocol used in this study was designed to determine whether these treatments could reverse pathological deficits associated with diabetic peripheral neuropathy including loss of corneal nerves and function.

Our results clearly demonstrate that vascular and neural deficits including cornea function and nerve density that were present following chronic hyperlipidemia and hyperglycemia in a rodent model of type 2 diabetes are reversible following treatment with menhaden oil, $a-$ lipoic acid or enalapril and to a greater extent after treatment with their combination. Even though the study design did not allow for determining the relative time line for reversal and/or improvement of these endpoints they have shown that improvement and regeneration of cornea nerve function and density, respectively, can serve as a biomarker for recovery of other neuropathy endpoints. In addition, the three compounds used for treatment and found to be efficacious in these studies have been widely used by humans. Fish oil supplements have been used to improve cardiovascular health and have been shown to improve metabolic features associated with type 2 diabetes. ${ }^{19-21}$ a-Lipoic acid is made in mammals, including humans, naturally, and is essential for aerobic metabolism. It is also manufactured and is available as an over the counter dietary antioxidant supplement. After a four year study of treatment for mild-to-moderate diabetic distal symmetric sensorimotor polyneuropathy, $a$ lipoic acid was not found to significantly influence the primary composite endpoints but did cause a clinically meaningful improvement and prevention of progression of neuropathic impairments and was well tolerated. ${ }^{22}$ Enalapril is an angiotensin converting enzyme inhibitor and these inhibitors have been a widely prescribed treatment for hypertension and diabetic nephropathy. ${ }^{23-26}$ Malik, et al. demonstrated that the angiotensin converting enzyme inhibitor trandolapril improved peripheral neuropathy in normotensive patients with diabetes. $^{27}$

A useful biomarker for screening and determining what treatments may be successful for diabetic peripheral neuropathy should be amendable to wide application and be able to objectively demonstrate efficacy. Clinical studies showing regeneration of corneal nerves following pancreas and pancreas/kidney transplant and our pre-clinical studies demonstrating full recovery of corneal nerve density and significant improvement in corneal 
nerve sensitivity with combination therapy provide support that examination of corneal nerve function and structure are objective and predictive outcome measures for treatment of diabetic neuropathy. ${ }^{12,13}$ Moreover, our pre-clinical studies demonstrated that mono-therapy and to a greater extent combination therapy efficaciously improved cornea nerve sensitivity and density and also reversed vascular and neural deficits associated with diabetic peripheral neuropathy.

In our study rats were fed a high fat diet for 24 weeks and were hyperglycemic for 16 weeks prior to treatment. Diabetes induced decrease in vascular function and nerve conduction velocity occurs early in rodents followed by thermal hypoalgesia, decrease in intraepidermal nerve fibers and decrease in corneal nerve function and density. $3,9,28-30$ Therefore, our ability to demonstrate that combination therapy following chronic hyperlipidemia and hyperglycemia could reverse all these deficits whether they occur early or later in duration of diabetes and that improvement of corneal nerve sensitivity and nerve density could predict these outcomes provides proof of concept that determination of corneal nerve function and structure may be the sensitive marker needed to monitor progression and treatment of diabetic peripheral neuropathy.

We recently introduced impairment of cornea sensitivity to a hypertonic solution as an objective marker for diabetic peripheral neuropathy. ${ }^{5}$ In that study we demonstrated that loss of corneal nerves in the sub-epithelial layer had a significant degree of correlation with the loss of corneal sensitivity. Impaired corneal sensitivity to a hypertonic solution also significantly correlated with the conventional diabetic neuropathy endpoints of slowing of motor and sensory nerve conduction velocity, thermal hypoalgesia and decrease in epidermal nerve fiber density. ${ }^{5}$ In this study, we show that improvement of corneal sensitivity to a hypertonic solution impaired by chronic diabetes is accompanied by regeneration of subepithelial corneal nerves. We observed minimal improvement when the chronically diabetic rats were treated with a-lipoic acid or enalapril alone but a significant improvement was achieved by treating diabetic rats with menhaden oil alone and to a greater extent the combination of menhaden oil, a-lipoic acid and enalapril.

Mechanisms contributing to neural dysfunction in diabetes that may be improved by these treatments would likely be oxidative and inflammatory stress. Our studies demonstrated that these treatments, with combination therapy being most efficacious, reduced serum levels of free fatty acids, triglycerides and cholesterol and thiobarbituric acid reactive substances, a marker of oxidative stress. It is unknown how altered lipid levels may contribute to diabetic neuropathy but diet-induced obese rats have been shown to have sensory neuropathy comparable to diabetes. ${ }^{28}$ The mechanism responsible for the improvement of serum lipids by the mono-therapies and to greater extent combination therapy is also unknown but would seem to be due in part to improvement of oxidative and inflammatory stress. It is known that the metabolites of $n-3$ polyunsaturated fatty acids have anti-inflammatory and neuroprotective properties. ${ }^{31-33}$ We have previously shown that enalapril and a-lipoic acid reduce markers of oxidative stress in blood vessels and serum of diabetic rats. ${ }^{34,35}$ We have also shown that enriching diets of diabetic rodents with menhaden oil reduces the n- 6 to $n-3$ fatty acid ratio and increases production of the docosahexaenoic metabolite resolvin D1. ${ }^{2,18}$ Additionally, we have shown that direct treatment of a mouse model of type 2 diabetes with 
daily injections of resolvin D1 improved diabetic peripheral neuropathy including intraepidermal nerve fiber density and density of sub-epithelial corneal nerve fibers and increased neurite outgrowth in cultured dorsal root ganglion neurons. ${ }^{18}$

In summary, this study has shown that treating type 2 diabetic rats following chronic hyperlipidemia and hyperglycemia with either menhaden oil, a-lipoic acid or enalapril or to a greater extent their combination can induce corneal nerve regeneration and recovery of sensitivity. Furthermore, treatments with combination therapy overall being the most efficacious also restored other endpoints associated with diabetic neuropathy as well as improved vascular reactivity of epineurial arterioles. We propose that clinically combination therapy will provide the most effective approach for treating diabetic neuropathy and that analysis of corneal sensitivity and cornea nerve density of the sub-epithelial layer may be a useful biomarker for evaluating recovery.

\section{Supplementary Material}

Refer to Web version on PubMed Central for supplementary material.

\section{Acknowledgments}

Conflicts of Interest and Source of Funding:

This material is based upon work supported in part by the Department of Veterans Affairs, Veterans Health Administration, Office of Research and Development, Rehabilitation Research and Development (RX000889-04; MAY) and Iowa City VA Center of Excellence for the Prevention and Treatment of Visual Loss: C9251-C (RHK), and by National Institute of Diabetes and Digestive and Kidney Diseases Grant DK107339 from NIH (MAY). The content of this manuscript are new and solely the responsibility of the authors and do not necessarily represent the official views of the granting agencies. R Kardon: cofounder of FaceX LLC. For the remaining authors none were declared.

\section{References}

1. Davidson EP, Holmes A, Coppey LJ, et al. Effect of combination therapy consisting of enalapril, alipoic acid, and menhaden oil on diabetic neuropathy in a high fat/low dose streptozotocin treated rat. Eur J Pharmacol. 2015; 765:258-267. [PubMed: 26291662]

2. Coppey LJ, Holmes A, Davidson EP, et al. Partial replacement with menhaden oil improves peripheral neuropathy in high-fat-fed low-dose streptozotocin type 2 diabetic rat. J Nutr Metab. 2012; 2012:950517. [PubMed: 22988492]

3. Davidson EP, Coppey LJ, Holmes A, et al. Effect of treatment of high fat fed/low dose streptozotocin-diabetic rats with Ilepatril on vascular and neural complications. Eur J Pharmacol. 2011; 668:497-506. [PubMed: 21816138]

4. Davidson EP, Coppey LJ, Holmes A, et al. Changes in corneal innervation and sensitivity and acetylcholine-mediated vascular relaxation of the posterior ciliary artery in a type 2 diabetic rat. Invest Ophthalmol Vis Sci. 2012; 53:1182-1187. [PubMed: 22273725]

5. Yorek MS, Davidson EP, Poolman P, et al. Corneal Sensitivity to Hyperosmolar Eye Drops: A Novel Behavioral Assay to Assess Diabetic Peripheral Neuropathy. Invest Ophthalmol Vis Sci. 2016; 57:2412-2419. [PubMed: 27145474]

6. Obrosova IG, Li F, Abatan OI, et al. Role of poly(ADP-ribose) polymerase activation in diabetic neuropathy. Diabetes. 2004; 53:711-720. [PubMed: 14988256]

7. Mihara M, Uchiyama M, Fukuzama K. Thiobarbituric acid value of fresh homogenate of rat as a parameter of lipid peroxidation in aging, $\mathrm{CCl} 4$ intoxication, and vitamin E deficiency. Biochem Med. 1980; 23:302-311. [PubMed: 7417237] 
8. Siman CM, Eriksson UJ. Vitamin C supplementation of the maternal diet reduces the rate of malformation in the offspring of diabetic rats. Diabetologia. 1997; 40:1416-1424. [PubMed: 9447949]

9. Coppey LJ, Davidson EP, Dunlap JA, et al. Slowing of motor nerve conduction velocity in streptozotocin-induced diabetic rats is preceded by impaired vasodilation in arterioles that overlie the sciatic nerve. Int J Exp Diabetes Res. 2000; 1:131-143. [PubMed: 11469397]

10. Hossain P, Sachdev A, Malik RA. Early detection of diabetic peripheral neuropathy with corneal confocal microscopy. Lancet. 2005; 366:1340-1343. [PubMed: 16226599]

11. Pritchard N, Edwards K, Vagenas D, et al. Corneal sensitivity as an ophthalmic marker of diabetic neuropathy. Optom Vis Sci. 2010; 87:1003-1008. [PubMed: 21037498]

12. Mehra S, Tavakoli M, Kallinikos PA, et al. Corneal confocal microscopy detects early nerve regeneration after pancreas transplantation in patients with type 1 diabetes. Diabetes Care. 2007; 30:2608-2612. [PubMed: 17623821]

13. Tavakoli M, Mitu-Pretorian M, Petropoulos IN, et al. Corneal confocal microscopy detects early nerve regeneration in diabetic neuropathy after simultaneous pancreas and kidney transplantation. Diabetes. 2013; 62:254-260. [PubMed: 23002037]

14. Gopinath S, Ganesh BA, Manoj K, et al. 2 year followup of patients with diabetes mellitus nephropathy showing albuminuria reversal following angiotensin converting enzyme inhibitors. Indian J Endocrinol Met. 2012; 16:S447-S449.

15. McIlduff CE, Rutkove SB. Critical appraisal of the use of alpha lipoic acid (thioctic acid) in the treatment of symptomatic diabetic polyneuropathy. Ther Clin Risk Manag. 2011; 7:377-385. [PubMed: 21941444]

16. Papanas N, Ziegler D. Efficacy of a-lipoic acid in diabetic neuropathy. Expert Opin Pharmacother. 2014; 15:2721-2731. [PubMed: 25381809]

17. Rudkowska I. Fish oils for cardiovascular disease: Impact on diabetes. Maturitas. 2010; 67:25-28. [PubMed: 20493648]

18. Shevalye H, Yorek MS, Coppey LJ, et al. Effect of enriching the diet with menhaden oil or daily treatment with resolvin D1 on neuropathy in a mouse model of type 2 diabetes. J Neurophysiol. 2015; 114:199-208. [PubMed: 25925322]

19. De Leiris J, De Lorgeril M, Boucher F. Fish oil and heart health. J Cardiovasc Pharmacol. 2009; 54:378-384. [PubMed: 19568180]

20. Jelinek D, Castillo JJ, Arora SL, et al. A high-fat diet supplemented with fish oil improves metabolic features associated with type 2 diabetes. Nutrition. 2013; 29:1159-1165. [PubMed: 23756282]

21. Mozaffarian D, Wu JHY. Omega-3 fatty acids and cardiovascular disease: effects on risk factors, molecular pathways, and clinical events. J Am Coll Cardiol. 2011; 58:2047-2067. [PubMed: 22051327]

22. Ziegler D, Low PA, Litchy WJ, et al. Efficacy and safety of antioxidant treatment with a-lipoic acid over 4 years in diabetic polyneuropathy: the NATHAN 1 trial. Diabetes Care. 2011; 34:20542060. [PubMed: 21775755]

23. Barnett A. Prevention of loss of renal function over time in patients with diabetic nephropathy. Am J Med. 2006; 119:S40-S47. [PubMed: 16563947]

24. Hilleman DE. Role of angiotensin-converting-enzyme inhibitors in the treatment of hypertension. Am J Health Syst Pharm. 2000; 57:S8-S11. [PubMed: 11030017]

25. Mogensen CE. New concepts in blood pressure-lowering management in diabetic patients: the case for early ACE inhibitor combination therapy with diuretics. J Hum Hypertens. 2005; 19:S15-S20. [PubMed: 16075028]

26. Ruggenenti P, Cravedi P, Remuzzi G. The RAAS in the pathogenesis and treatment of diabetic nephropathy. Nat Rev Nephrol. 2010; 6:319-330. [PubMed: 20440277]

27. Malik RA, Williamson S, Abbott C, et al. Effect of angiotensin-converting-enzyme (ACE) inhibitor trandolapril on human diabetic neuropathy: randomized double-blind controlled trial. Lancet. 1998; 352:1978-1981. [PubMed: 9872248] 
28. Davidson EP, Coppey LJ, Kardon RH, et al. Differences and similarities in development of corneal nerve damage and peripheral neuropathy and in diet-induced obesity and type 2 diabetic rats. Invest Ophthalmol Vis Sci. 2014; 55:1222-1230. [PubMed: 24519423]

29. Yorek MA, Coppey LJ, Gellett JS, et al. Sensory nerve innervation of epineurial arterioles of the sciatic nerve containing calcitonin gene-related peptide: effect of streptozotocin-induced diabetes. Exp Diabesity Res. 2004; 5:187-193. [PubMed: 15512786]

30. Coppey LJ, Gellett JS, Yorek MA. Mediation of vascular relaxation in epineurial arterioles of the sciatic nerve: effect of diabetes in type 1 and type 2 diabetic rat models. Endothelium. 2003; 10:89-94. [PubMed: 12791516]

31. Ji RR, Xu ZZ, Strichartz G, et al. Emerging roles of resolvins in the resolution of inflammation and pain. Trends Neurosci. 2011; 34:599-609. [PubMed: 21963090]

32. Kohli P, Levy BD. Resolvins and protectins: mediating solutions to inflammation. Brit J Pharmacol. 2009; 158:960-971. [PubMed: 19594757]

33. Serhan CN, Hong S, Gronert K, et al. Resolvins: A family of bioactive products of omega-3 fatty acid transformation circuits initiated by aspirin treatment that counter proinflammation signals. J Exp Med. 2002; 196:1025-1037. [PubMed: 12391014]

34. Coppey LJ, Gellett JS, Davidson EP, et al. Effect of antioxidant treatment of streptozotocininduced diabetic rats on endoneurial blood flow, motor nerve conduction velocity, and vascular reactivity of epineurial arterioles of the sciatic nerve. Diabetes. 2001; 50:1927-1937. [PubMed: 11473057]

35. Coppey LJ, Davidson EP, Rinehart TW, et al. ACE inhibitor or angiotensin II receptor antagonist attenuates diabetic neuropathy in streptozotocin-induced diabetic rats. Diabetes. 2006; 55:341348. [PubMed: 16443766] 


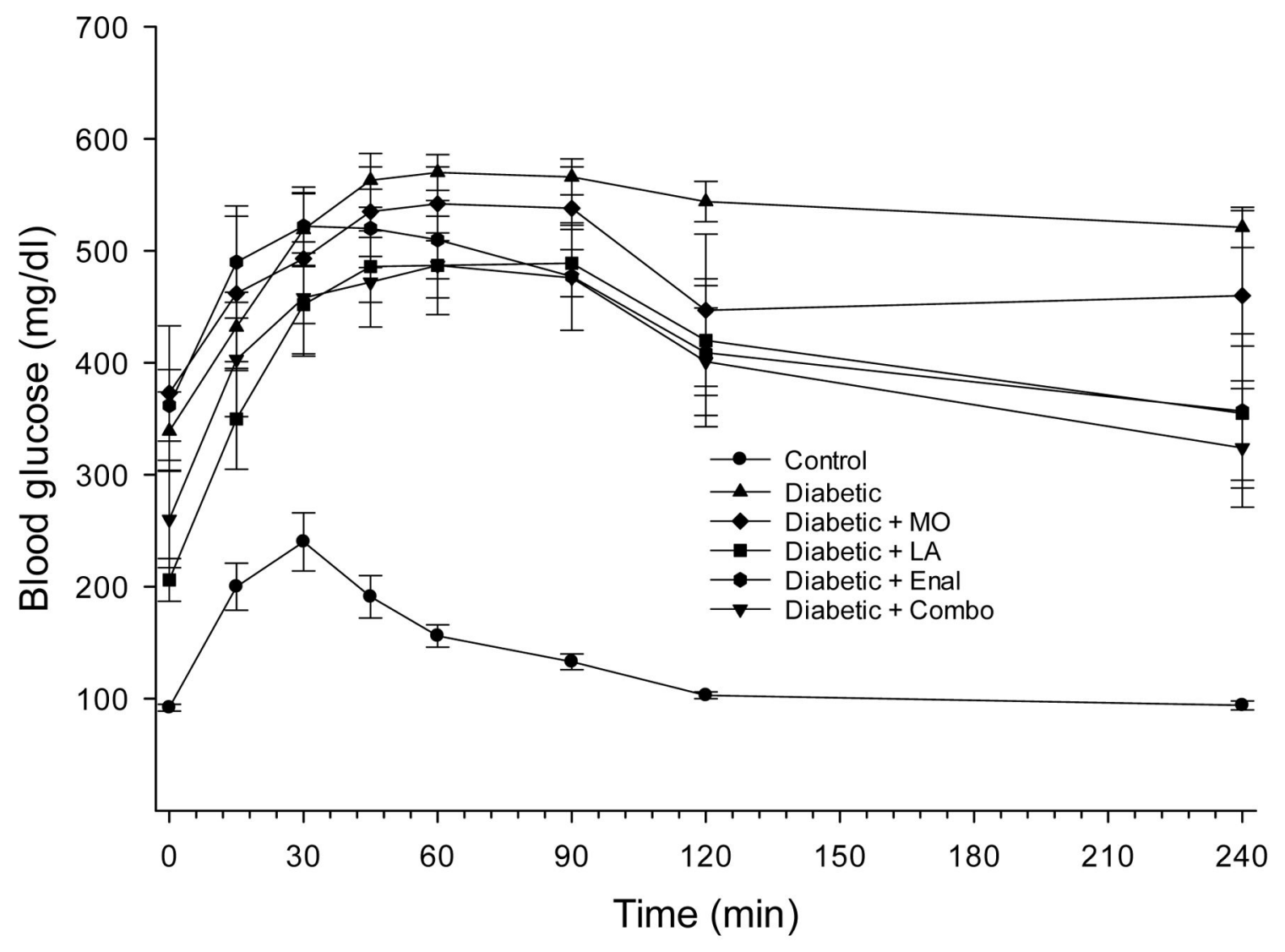

Figure 1. Glucose utilization curve for control rats, untreated type 2 diabetic rats and type 2 diabetic rats treated with menhaden oil, a-lipoic acid, enalapril or their combination

Fasting blood glucose at time 0 for control, untreated type 2 diabetic rats and type 2 diabetic rats treated with menhaden oil, a-lipoic acid, enalapril or their combination for early intervention was $92 \pm 3,339 \pm 35^{*}, 373 \pm 60^{*},+, 206 \pm 19^{*}, 362 \pm 32^{*}$ and $260 \pm 43^{*} \mathrm{mg} / \mathrm{dl}$, respectively and for late intervention was $94 \pm 3,325 \pm 24^{*}, 355 \pm 51^{*}, 250 \pm 27^{*}, 324 \pm 36^{*}$ and $280 \pm 43^{*} \mathrm{mg} / \mathrm{dl}$, respectively (* $\mathrm{p}<0.05$, compared to control rats). Data are the mean \pm S.E.M. The area under the curve (AUC) was significantly different, $\mathrm{p}<0.05$ (impaired), for untreated type 2 diabetic rats and type 2 diabetic rats treated with menhaden oil, a-lipoic acid, enalapril or the combination vs. control rats. The number of rats in each group was the same as shown in Table 1. 


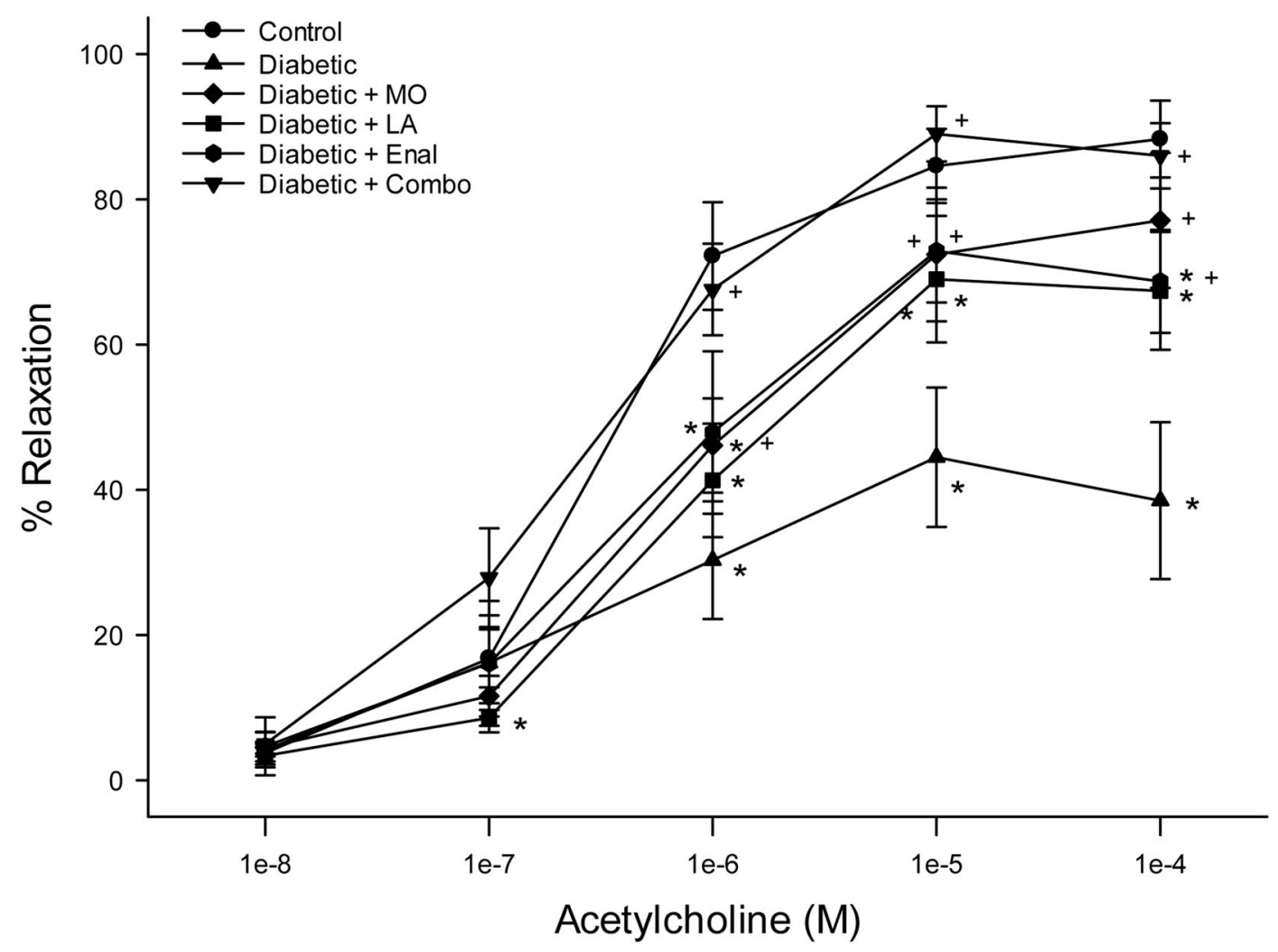

Figure 2. Effect of treating diabetic rats with menhaden oil, a-lipoic acid, enalapril or their combination on vascular relaxation by acetylcholine in epineurial arterioles

Vascular relaxation to acetylcholine was determined as described in the legend of Figure 3. The number of rats in each group was the same as shown in Table 1. Data are presented as the mean of $\%$ relaxation \pm S.E.M. $* p<0.05$ compared to control rats $; p<0.05$ compared to diabetic rats. 


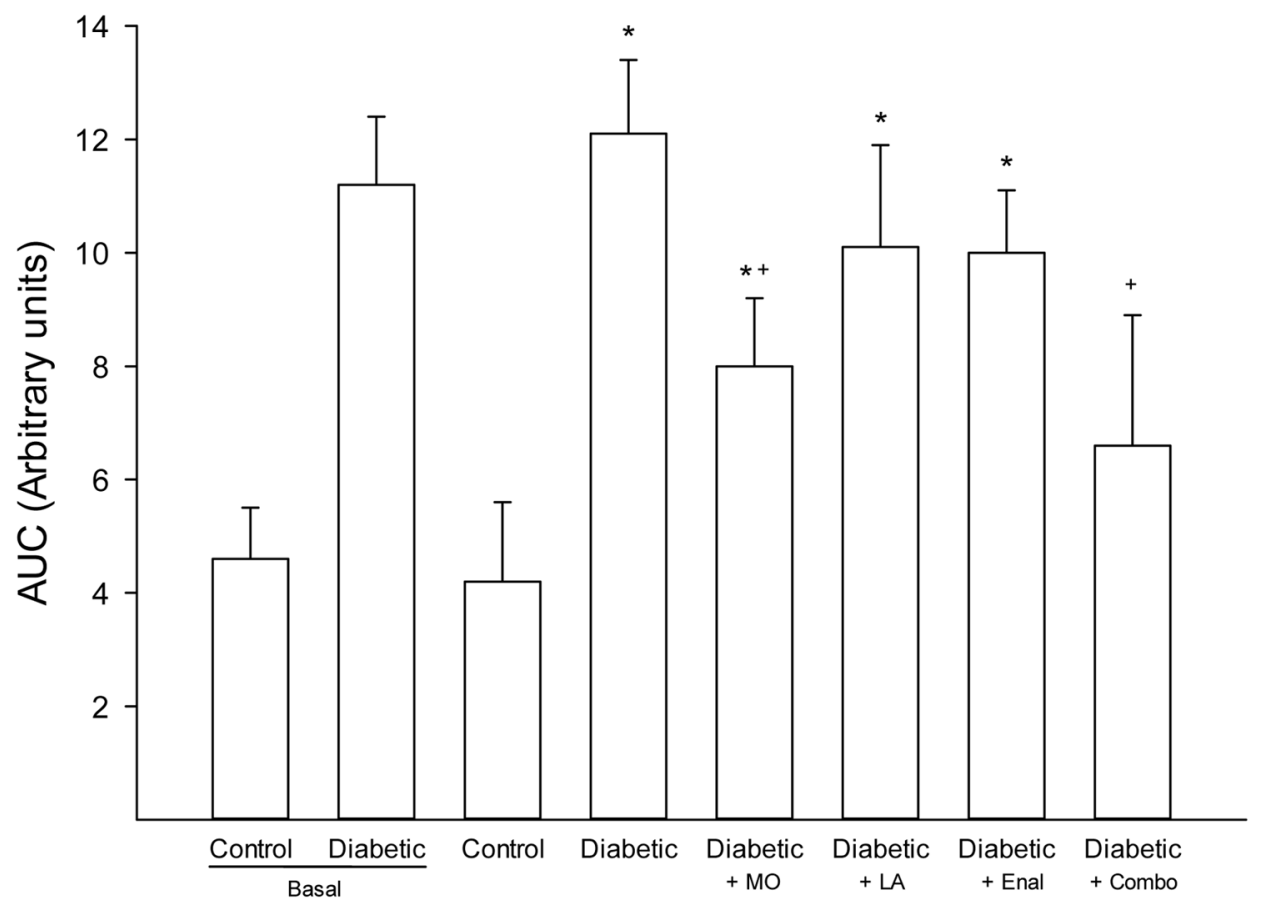

Figure 3. Effect of treating diabetic rats with menhaden oil, a-lipoic acid, enalapril or their combination on corneal reactivity to a hypertonic solution

Corneal reactivity was determined and quantified as described in the Methods section. The data set labeled as basal was obtained from control and diabetic rats examined prior to initiation of treatments $(n=6)$. The number of rats for the treatment groups was the same as shown in Table 1. Data are presented as area under the curve. A larger area under the curve occurs with little squinting and lack of narrowing of the inter-palpebral area due to loss of corneal sensation in response to a hypertonic saline drop. ${ }^{*} \mathrm{p}<0.05$ compared to control rats; $+\mathrm{p}<0.05$ compared to diabetic rats. 


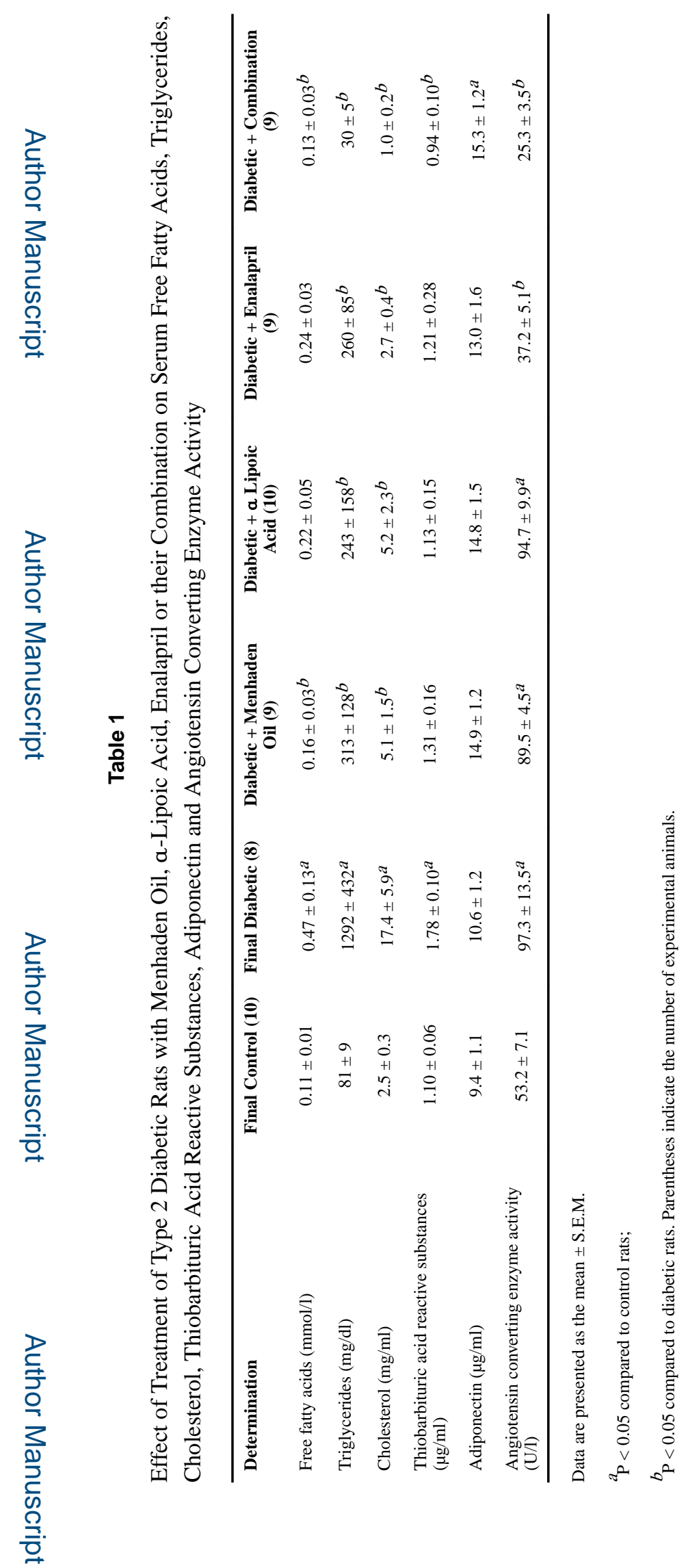

Cornea. Author manuscript; available in PMC 2018 June 01. 


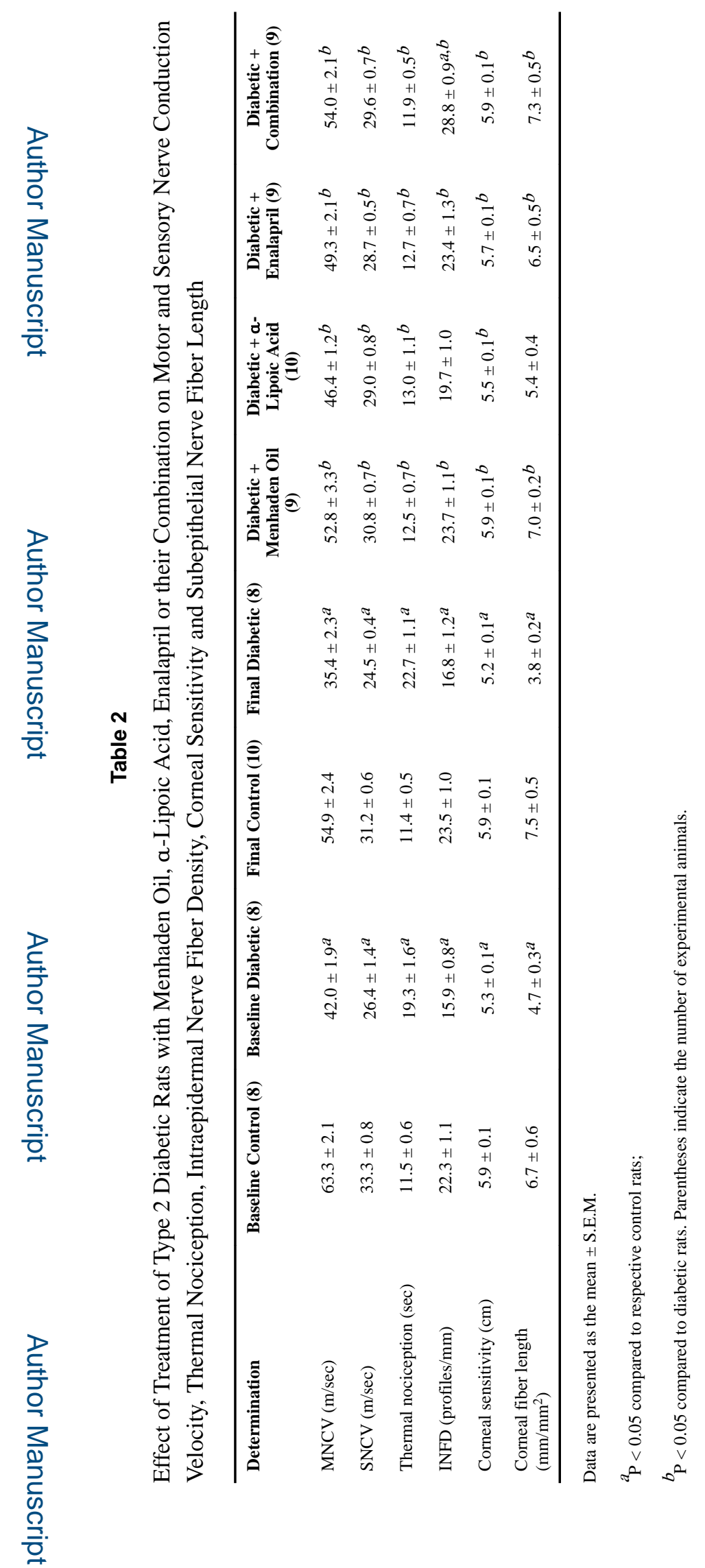

Cornea. Author manuscript; available in PMC 2018 June 01. 